\title{
Philosophiques
}

\section{Magistrats et gens de bien}

\section{Nicolas Tavaglione}

Volume 32, numéro 2, automne 2005

URI : https://id.erudit.org/iderudit/011869ar

DOI : https://doi.org/10.7202/011869ar

Aller au sommaire du numéro

\section{Éditeur(s)}

Société de philosophie du Québec

\section{ISSN}

0316-2923 (imprimé)

1492-1391 (numérique)

Découvrir la revue

\section{Citer cet article}

Tavaglione, N. (2005). Magistrats et gens de bien. Philosophiques, 32(2), 295-317. https://doi.org/10.7202/011869ar

\section{Résumé de l'article}

Je propose ici un argument " dilemmatique " en faveur de l'anti-paternalisme libéral. Soit il est vrai, comme le soutiennent les kantiens, que les règles impersonnelles, impartiales et universelles jouissent d'une priorité éthique ; soit il est vrai, comme le soutiennent des communautariens comme MacIntyre, que de telles règles ne jouissent pas d'une priorité éthique. Si les kantiens ont raison, alors - comme le veut la sagesse conventionnelle des manuels de philosophie politique - la neutralité de l'Etat à l'endroit des conceptions du bien est justifiée. Et si les communautariens ont raison, alors - étonnamment la neutralité de l'Etat est aussi justifiée. Donc la neutralité de l'Etat est justifiée. La première prémisse étant tautologique, elle s'impose d'elle-même. La seconde prémisse, établissant un lien entre priorité des règles universelles et neutralité de l'Etat, est trop connue pour retenir ici notre attention. La troisième prémisse, en revanche, établissant un lien entre la non-priorité des règles universelles et la neutralité de l'Etat, sonne comme un paradoxe. C'est donc sur elle que portera l'examen.
Ce document est protégé par la loi sur le droit d'auteur. L’utilisation des services d'Érudit (y compris la reproduction) est assujettie à sa politique d'utilisation que vous pouvez consulter en ligne.

https://apropos.erudit.org/fr/usagers/politique-dutilisation/ 


\title{
Magistrats et gens de bien
}

\author{
NICOLAS TAVAGLIONE \\ Université de Genève \\ Département de science politique \\ nicolas.tavaglione@politic.unige.ch
}

\begin{abstract}
RÉSUMÉ. - Je propose ici un argument «dilemmatique» en faveur de l'antipaternalisme libéral. Soit il est vrai, comme le soutiennent les kantiens, que les règles impersonnelles, impartiales et universelles jouissent d'une priorité éthique; soit il est vrai, comme le soutiennent des communautariens comme Maclntyre, que de telles règles ne jouissent pas d'une priorité éthique. Si les kantiens ont raison, alors - comme le veut la sagesse conventionnelle des manuels de philosophie politique - la neutralité de l'Etat à l'endroit des conceptions du bien est justifiée. Et si les communautariens ont raison, alors - étonnamment - la neutralité de l'Etat est aussi justifiée. Donc la neutralité de l'Etat est justifiée. La première prémisse étant tautologique, elle s'impose d'elle-même. La seconde prémisse, établissant un lien entre priorité des règles universelles et neutralité de l'Etat, est trop connue pour retenir ici notre attention. La troisième prémisse, en revanche, établissant un lien entre la non-priorité des règles universelles et la neutralité de l'Etat, sonne comme un paradoxe. C'est donc sur elle que portera l'examen.
\end{abstract}

ABSTRACT. - I shall offer here a "dilemmatic" argument for liberal antipaternalism. Either it is the case - as Kantians maintain - that impersonal, impartial and universal rules enjoy ethical priority; or it is the case - as Communitarians like Maclntyre maintain - that universal rules do not enjoy any such priority. If Kantians are right, then - as suggested by the conventional wisdom of textbooks in political philosophy - State-neutrality towards conceptions of the good is justified. And if Communitarians are right, then - surprisingly - State-neutrality is justified too. Therefore State-neutrality is justified. The first premiss is tautological, and needs no special study. The second premiss, linking the priority of universal rules to State-neutrality, is too well known to deserve our attention. But the third premiss, linking the non-priority of universal rules to State-neutrality, sounds like a paradox. The paper thus focuses on that third controversial premiss.

Il n'est pas possible [...] de penser que les hommes doivent donner au magistrat le pouvoir de choisir à leur place la voie qui mène au salut.

LOCKE $(1667$, p. 109)

\section{Introduction'}

Nous sommes habitués depuis au moins trente ans, sur les conseils du "premier Rawls» de la Théorie de la justice, à croire en l'existence d'un lien

1. Je remercie le Fonds national (suisse) de la recherche scientifique, grâce auquel j'ai pu commencer cet article, et le Département de science politique de l’Université de Genève, grâce 
privilégié entre le libéralisme et la morale kantienne ${ }^{2}$. Le raisonnement à l'appui de cette thèse peut être résumé ainsi. Le libéralisme politique contemporain revendique la priorité du juste sur le bien. Or la justification la plus sérieuse de cette priorité est à trouver dans les écrits moraux de Kant, lesquels insistent sur l'autonomie rationnelle des agents moraux. La valeur morale impliquant dans cette optique l'autonomie rationnelle, il s'ensuit qu'on ne peut promouvoir ou maximiser la "somme de valeur morale» réalisée dans le monde en imposant aux agents moraux des fins hétéronomes. Le juste, à savoir un ensemble de règles destinées à gouverner les comportements individuels et dignes de recevoir l'assentiment rationnel de tous ceux et celles qui tombent sous leur juridiction, doit avoir en morale la priorité sur le bien, à savoir la réalisation d'une vie bonne ${ }^{3}$. La poursuite du bien doit être limitée par les contraintes du juste, pour que chacun puisse poursuivre librement son bien dans le cadre de la justice. Sur un plan plus clairement politique, la priorité du juste se traduit traditionnellement, dans le libéralisme, par le souci de garantir la neutralité de l'État.

Nombre de critiques communautariens du libéralisme s'en sont donc pris naturellement à la priorité kantienne du juste, suivant les contours de deux arguments principaux. (i) Il y a d'abord l'assaut direct contre $\mathrm{Kant}^{4}$. Le libéralisme suppose la priorité du juste sur le bien; or cette priorité est intenable, car les règles du juste imposent à nos réflexions éthiques une généralité, une impartialité et une abstraction qui ne sont nullement nécessaires. Puisqu'on ne peut admettre la priorité du juste dans les affaires éthiques en général, on ne peut l'admettre dans les affaires politiques, et le libéralisme est sans fondement. Resquiet in pace. MacIntyre (1981) illustre à merveille cette stratégie: l'insuffisance de la théorie morale kantienne entraîne à ses yeux, sans autre forme de procès, celle du libéralisme. (ii) Il y a ensuite une attaque indirecte, qui se présente comme suit. On sait que les libéraux con-

auquel je peux le terminer. De nombreuses personnes - philosophes, historiennes ou politologues - m'ont fait bénéficier de leurs précieux commentaires sur des versions primitives du texte: Pierre Allan, Bernard Baertschi, Axel Gosseries, Alexis Keller, Philip Keller, Elise Labrèque, Kevin Mulligan, Marc Ruegger, Philippe Van Parijs, Sandra Froidevaux, Emma Tiefenbach et Victoria Tschumi, ainsi que deux lecteurs anonymes de la revue Philosophiques. Je n'ai pu tenir compte de toutes leurs suggestions et objections, mais elles m'ont épargné de nombreuses erreurs. Enfin, je dois la plus grande gratitude à Markus Haller et Emmanuelle Joz-Roland - dont l'attention et la conversation m'ont été d'un apport inestimable.

2. Pour un exemple récent de cette croyance, en français, cf. Renaut (1999), p. ex. : 29 sqq.

3. Cf. p. ex. Kant (1788), I, 2, ch. 1, p. 151: «Si l'on admet, avant la loi morale, comme principe déterminant de la volonté, un objet quelconque présenté sous le nom d'un bien, et qu'on en dérive ensuite le principe pratique suprême, celui-ci introduirait toujours alors de l'hétéronomie et expulserait le principe moral.»

4. En employant le mot «Kant », je désigne moins le philosophe historique que le modèle simplifié, et peut-être infidèle à la «réalité » d'une œuvre complexe, qui circule dans la littérature moyenne sur le libéralisme. Les objections exégétiques auront donc peu de poids dans mon propos, qui n'est en rien exégétique. 
temporains - dans la lignée des arguments classiques en faveur de la séparation entre l'État et l'Église - insistent sur le fait du pluralisme raisonnable: il existe, dans les sociétés occidentales modernes, un pluralisme irréductible des conceptions religieuses ou éthiques de la vie bonne. On sait également que la tradition libérale revendique un principe de légitimité impartial: une institution politique est légitime quand elle reçoit une justification que toutes les personnes concernées peuvent raisonnablement accepter. Pluralisme raisonnable et principe de légitimité entraînent la conclusion suivante: il ne faut pas justifier les institutions politiques sur la base d'une doctrine morale particulière - sans quoi, en raison du pluralisme raisonnable, on viole immanquablement le principe de légitimité. Les institutions politiques doivent donc recevoir une justification publique, moralement neutre, plutôt qu'une justification privée, moralement partisane. Se dessine ainsi le modèle de libéralisme le plus en vogue aujourd'hui: le libéralisme de la raison publique, qui réclame des justifications moralement neutres ${ }^{5}$. Mais ce dernier semble devoir s'empaler sur une des cornes du dilemme suivant: a) soit le libéralisme politique n'a aucun contenu moral, mais il semble alors manquer à la fois de profondeur, de pertinence éthique et d'efficace politique; $b$ ) soit il a un contenu moral, mais il viole alors le principe de légitimité susmentionné et semble inconciliable avec le pluralisme (Chen, 1998). Si le libéralisme politique est bien tributaire de la priorité kantienne du juste, alors il viole le principe de légitimité impartial. Il existe en effet des arguments raisonnables, comme en atteste l'existence des communautariens, pour être en désaccord avec la morale kantienne. Mais si le libéralisme politique viole le principe de légitimité impartial, il semble violer du même coup l'autonomie des non-kantiens. Et il s'avère incapable de respecter ses propres exigences.

Face à cette double critique, les réactions peuvent être diverses. On peut, comme le «second Rawls» de Libéralisme politique, tenter de "dékantiser» le libéralisme. Mais on s'expose à la première corne du dilemme. Ou on peut, comme John Skorupski (1999) par exemple, revendiquer le «perfectionnisme» du libéralisme comme une qualité, et vouloir le fonder sur l'idéal d'autonomie. Mais on se heurte toujours à la seconde corne du dilemme, privant le libéralisme d'une de ses vertus cardinales - d'un point de vue moral comme épistémologique -, à savoir l'impartialité à l'endroit des Weltanschaunngen concurrentes. Les deux types de plaidoyer semblent peu prometteurs. C'est pourquoi j'aimerais explorer une défense alternative du libéralisme politique. Soit MacIntyre (1981). Il soutient qu'il est déraisonnable d'accepter, en éthique, la primauté des règles générales, impersonnelles et abstraites - et donc d'admettre la priorité du juste. Je voudrais montrer que, si MacIntyre a raison, alors il existe un excellent argument en faveur de la neutralité de l'État. Plus précisément, il existe un excellent argument pour rejeter le paternalisme moral - i.e. l'idée selon laquelle il devrait, et donc il

5. Cf. entre autres, Gaus, 1999; Macedo, 1991; Nagel 1987. 
pourrait, être du ressort de la loi de promouvoir le bien. Or le rejet du paternalisme moral est typiquement libéral. Si la priorité du juste est intenable en éthique, et précisément parce qu'elle est intenable, il existe une raison d'être libéral et de revendiquer la neutralité de l'État - et donc une version politique de la priorité du juste sur le bien.

Une telle ligne de défense se distingue par quatre qualités. Primo, elle prend au sérieux les arguments hostiles à la priorité éthique du juste sur le bien, faisant ainsi une concession de taille à l'adversaire - ce qui est toujours un bon début. Secundo, elle dissocie libéralisme et kantisme en montrant qu'on peut obtenir des conclusions libérales en partant de prémisses anti-kantiennes - et sauve ainsi les amis du libéralisme d'une énervante crampe mentale. Tertio, elle nous libère du dilemme présenté plus haut. Si les communautariens comme MacIntyre ont raison (en éthique), alors les libéraux ont raison (en politique). Mais si Kant a raison, il semblerait que les libéraux aient aussi raison (à en croire les communautariens). Il apparaît ainsi que le libéralisme peut avoir plusieurs contenus moraux contradictoires. Si tel est le cas, il peut «avoir du contenu» d'un point de vue moral, et échapper à la première corne du dilemme; mais ce contenu étant pluriel, il échappe au reproche de perfectionnisme partisan animant la seconde corne. Nous obtenons ainsi une nouvelle bonne raison de penser, comme Rawls (1993) le soutenait, que le libéralisme politique, et sa neutralité, est une théorie politique susceptible de consensus par recoupement. Quarto, elle nous débarrasse d'une prémisse cachée, mais fondamentale, de l'argument de MacIntyre: si P est inacceptable en éthique, alors $\mathrm{P}$ est inacceptable en politique - en l'occurrence: si la priorité du juste sur le bien est intenable en éthique, alors elle est intenable en politique (exit la neutralité de l'État). Il y aurait ainsi continuité entre éthique et politique. L'argument développé ici donne une preuve par l'acte que cette continuité est illusoire. Et il nous offre un motif supplémentaire de méfiance à l'égard du moralisme - défini comme le projet de soumettre la philosophie politique aux mêmes critères de réussite que la philosophie morale.

Je procéderai comme suit. D'abord, je caractériserai assez brièvement ce que je nommerai le point de vue (légal) des magistrats (section 3). Ensuite, je montrerai que le point de vue (éthique) des gens de bien diffère du premier sous des aspects importants, si bien qu'on ne doit pas les confondre. Ils diffèrent en ceci, notamment, que le premier exclut que le jugement soit influencé par des informations personnelles et particulières, tandis que le second l'exige parfois (souvent). Je me baserai pour étayer cette affirmation sur des arguments tirés de la littérature contemporaine dite «anti-théorique » - et dans une large mesure anti-kantienne - en éthique (section 4). Enfin, je présenterai les implications de la distinction ainsi tracée sur la question du paternalisme moral compris ici comme l'idée selon laquelle la loi doit se charger de promouvoir la vie bonne ${ }^{6}$. Ces implications seront bien entendu négatives: il serait dérai-

6. Cf. Kekes, 1997. 
sonnable, d'un point de vue éthique, de confier à la loi la promotion du «bien ", parce que le point de vue des magistrats ne permet pas de parvenir à des jugements éthiques appropriés (section 5). Le résultat majeur de la «démonstration ", si toutefois elle est solide, étant d'attirer l'attention sur une vérité assez simple: on peut être libéral même si l'on rejette la priorité kantienne du juste en éthique, ou, bien plus, parce qu'on la rejette (section 6).

Il va donc s'agir de comparer, pour les contraster, les points de vue éthique et légal. Qu'est-ce, avant tout, qu'un point de vue?

\section{Le concept de point de vue}

Définissons un point de vue de type F comme celui dont l'adoption nous permet de parvenir à des jugements de type F appropriés. Le " point de vue médical », ainsi, est celui qu'il faut adopter pour parvenir à des jugements médicaux appropriés. Supposons qu'ayant consulté un généraliste, j’entende le diagnostic suivant: "Vous souffrez d'un dysfonctionnement de la rate parce que vous avez trop ardemment convoité la femme d'autrui. Revenez dans le droit chemin, et votre organe retrouvera la santé. » Je peux inférer que le médecin m'a examiné d'un point de vue non pas médical -, mais religieux. Ou supposons que j'affirme: "Je n'aime pas ce roman, parce qu'il est trop grand pour entrer dans mes rayonnages. " Dira-t-on que j'ai adopté le point de vue de l'esthète? Il est plus probable, au contraire, que j'ai évalué le livre en question du point de vue du libraire ou de l'homme d'intérieur. Supposons enfin qu'un juge prononce la sentence suivante: "Vous êtes innocente, parce que vous êtes belle. » L'homme a-t-il adopté le point de vue légal ? Plus raisonnablement, il faudrait penser qu'il a délibéré du point de vue de l'esthète ou de «l'amant ". Ces trois exemples illustrent des cas de jugement inapproprié.

Notons qu'un jugement peut être approprié et faux, ainsi qu'inapproprié et vrai. Le premier cas est illustré par l'énoncé suivant, prononcé par un médecin: "Pour guérir de vos migraines, il vous faut porter des lunettes à double foyer ", alors que j'ai une tumeur au cerveau dont la guérison requiert une chimiothérapie. Le second cas est illustré par l'énoncé suivant, toujours prononcé par un médecin: "Il pleut », alors que je lui demande pourquoi j'ai mal au crâne et qu'il pleut effectivement. La relation "être approprié à » est donc différente de la relation «être vrai », en ce sens que la seconde se rapporte à une représentation du monde extérieur tandis que la première se rapporte à une réponse à une question. Un jugement est approprié, par conséquent, quand il est de nature à répondre à la question posée. Il ne faut donc pas confondre la relation d'à propos qui nous intéresse ici avec celle qui intervient en philosophie des émotions - les critères d'à propos pertinents concernant alors la relation entre l'émotion, son objet extérieur et la justification de la première par le second.

La sélection du point de vue doit donc être déterminée par la question posée. Pourquoi le jugement légal: "Vous êtes innocente, parce que vous êtes belle» est-il inapproprié ? Parce qu'il fait intervenir des considérations - en 
l'occurrence une prémisse «érotique » - qui devraient n'avoir aucune influence sur la question, par exemple: "Susanne est-elle coupable du meurtre de Max?". La base d'information des jugements légaux exclut ainsi les considérations érotiques. L'adoption d'un point de vue de type F, partant, consiste à choisir la base d'information propre aux jugements de type $\mathrm{F}$, base d'information constituée d'un ensemble de contraintes pesant sur le choix des informations appelées à déterminer le jugement.

Venons-en aux rapports entre éthique et loi. La question que je voudrais poser est la suivante: le point de vue (éthique) des gens de bien et le point de vue (légal) des magistrats sont-ils isomorphes - i.e. les jugements éthique et légal sont-ils soumis aux mêmes contraintes?

\section{Le point de vue légal des magistrats}

Penchons-nous d'abord sur le jugement légal. Ce dernier, notons-le d'entrée de jeu, n'est pas un jugement sur la loi. Lorsque je dis d'une loi qu'elle est dangereuse, je porte sur elle un jugement prudentiel, et non légal. Et lorsque, parlementaire ou simple citoyen, je dis d'une loi qu'elle mérite d'être adoptée, je porte sur elle un jugement législatif, et non légal. Le jugement légal qui nous intéressera est un jugement par la loi; plus précisément, il est la conclusion, sous la forme d'une proposition normative particulière, d'un syllogisme subsumant des circonstances particulières sous une loi générale. "Il est interdit d'accomplir des actes de type $\mathrm{F}$; or l'action particulière $a$ est de type $\mathrm{F}$; donc il est interdit d'accomplir $a$ » : telle est la forme la plus simple du «syllogisme normatif », lequel devient légal si sa majeure contient une règle juridique. Un jugement légal est justifié s'il applique correctement une règle juridique aux circonstances particulières pour en déduire une norme elle aussi particulière. Dans les jugements légaux, par conséquent, la justification a pour condition nécessaire et suffisante la dérivation à partir d'une loi.

Qu'est-ce qu'une loi ? La question est notoirement difficile, et peut-être pourrait-on dire de la loi ce que Robert Musil disait de la théologie: "On ignore tout de la théologie, bien qu'on ne puisse nier qu'il existe toujours, effectivement, beaucoup d'églises ${ }^{7}$. On ignore tout de la loi; mais on ne peut nier qu'il existe beaucoup de tribunaux. J'aimerais ainsi me concentrer sur les jugements légaux allant chercher leurs prémisses normatives dans ces corps de lois dont l'existence est la moins sujette à discussion, parce qu'elle a des effets observables - i.e. qu'elle est effectivement appliquée, dans les tribunaux, par le juge, et dans la rue, par la maréchaussée.

Une loi est une règle juridique. Sur le plan «formel », en tant qu'elle est une règle, elle est une proposition normative universelle ${ }^{8}$. Sur le plan

7. Musil, 1956, 33.

8. L'universalité dont il est ici question est logique. Il s'ensuit qu'une loi doit être logiquement universelle, mais qu'elle peut l'être à l'égard d'une classe spécifique de cas: «Tous les jJuifs doivent porter l'étoile jaune». Dans ce dernier cas, elle n'est pas censée valoir pour tout le monde, mais pour tous les individus répondant à une description explicitement spécifiée dans la proposition normative. 
«matériel », en tant qu'elle est une règle juridique, elle s'inscrit dans un système juridique. Un système juridique est un ensemble coordonné et hiérarchisé de "règles de droit", appuyées par le monopole de la violence légitime, appliquées par les membres d'institutions idoines - les tribunaux - et profitant d'une "habitude générale d'obéissance ». Dès lors qu'ils atteignent un certain degré de complexité - que ce soit dans l'Athènes d'Aristote ou dans les démocraties libérales contemporaines -, les systèmes juridiques contiennent au moins deux types, grossièrement définis, de règles de droit. Les premières - les règles primaires - prescrivent inconditionnellement certaines actions ou abstentions aux individus qui tombent sous leur juridiction. Les secondes - les règles secondaires - établissent certaines procédures visant à diminuer l'indétermination et l'incertitude des premières en leur garantissant une reconnaissance, une interprétation et une application à la fois publiques et irrévocables ${ }^{9}$. Attardons-nous sur les règles primaires - qui nous intéressent au premier chef, puisqu'elles sont, des règles de droit qui constituent un système juridique, celles qui ressemblent le plus aux impératifs moraux et donc celles qui touchent de plus près aux rapports entre loi et éthique. De telles règles, nous pouvons dégager sept traits idéaux-typiques.

a) Elles sont générales. Nous savons déjà que les règles juridiques sont des propositions normatives universelles. Il faut ajouter qu'elles doivent pouvoir s'appliquer à une variété indéfinie de cas concrets dont les traits particuliers sont imprévisibles. Pour reprendre un exemple d'Aristote, une loi interdisant le meurtre ne peut spécifier toutes les "armes » mortelles possibles; de même, par exemple, elle ne peut spécifier toutes les circonstances concrètes rendant un meurtre " passionnel » et accordant à son auteur le bénéfice des circonstances atténuantes.

b) Partant, elles sont abstraites - i.e. elles ne mentionnent qu'une infime partie des traits de la situation concrète particulière à laquelle elles s'appliquent.

c) De même, elle sont impersonnelles - i.e. elles excluent les noms propres et elles ne mentionnent qu'une infime partie des traits de la personne concrète particulière à laquelle elles s'adressent et qui doit leur obéir.

d) Elles énoncent des prescriptions obligatoires, en ce sens qu'on doit y obéir, qu'on le veuille ou non ${ }^{10}$.

e) Ces "obligations" doivent jouir d'une reconnaissance publique, sans quoi elles ne peuvent effectivement réguler les comportements et nourrir

9. Pour plus de détails, cf. Hart (1961), chap. V; pour un survol des théories contemporaines du droit, cf. Kelly (1992: chap. 9 et 10) et Wacks (2005).

10. L'interdiction du meurtre est une prescription obligatoire: elle s'applique à moi indépendamment de mes désirs ou de mes croyances concernant son bien-fondé. Le cas des permissions est plus complexe (à cause, notamment, de cette simple question: doit-on compter au nombre des permissions légales les types d'actes que la loi autorise explicitement, ou faut-il y ajouter les types d'actes sur lesquels la loi demeure muette?); et, faute de place, j'en omets la discussion. 
des prévisions claires sur le comportement d'autrui. D'où on peut inférer que les lois doivent être stables, car, changent-elles trop souvent, et aucune prévision n'est possible; et qu'elles ne doivent pas être rétroactives, car je ne peux régler mes comportements sur des normes inexistantes.

f) Les règles juridiques doivent pouvoir conduire à des jugements univoques, sans quoi nous serions soumis à des conflits d'obligations rendant l'obéissance à la loi difficile ou impossible. Elles sont ainsi mutuellement compatibles, dans le meilleur des cas, et sinon, à tout le moins, gouvernées par une règle connexe de priorité. Disons ainsi, pour plus de concision, que les règles juridiques sont harmonieuses ${ }^{11}$.

g) De surcroît, puisqu'elles doivent être susceptibles de reconnaissance publique, elles doivent aussi pouvoir être explicites pour permettre l'expression publique des jugements, des décisions ou des interprétations autorisés par le système juridique.

Nous trouvons donc que les règles juridiques doivent être: $a$ ) générales; $b)$ abstraites; $c$ ) impersonnelles; $d$ ) obligatoires; $e$ ) publiques; $f$ ) harmonieuses; et g) explicites. La base d'information des jugements légaux est ainsi restreinte à des considérations normatives universelles, impersonnelles, obligatoires, publiques, harmonieuses et explicites (à tout le moins explicitables). Adopter le point de vue du magistrat sur une question pratique revient donc à n'accepter que des prémisses prescriptives satisfaisant les sept conditions qui précèdent. C'est ce que fait un juge quand il remplit correctement sa fonction - quand il joue bien son rôle ${ }^{12}$.

Quelques précisions sont ici nécessaires. On objectera que cette description du "point de vue du magistrat » trahit un puissant biais positiviste, et qu'il présente la fonction judiciaire comme un mécanisme déductif transformant les juges en automates. Ces derniers, en réalité, ne se contentent pas d'appliquer la loi : ils interprètent la loi existante, et, peut-être, en complétant les trous du code par la jurisprudence, créent de la loi nouvelle. Nous ne

11. Dans le droit positif, c'est bien connu, cette harmonie est imparfaite - et tout système de lois réel enveloppe des contradictions dues à l'histoire et aux implications imprévisibles des textes. Si bien, comme le fait remarquer un lecteur anonyme de Philosophiques, que «ce n'est guère un trait des règles juridiques d'être d'emblée harmonieuses. Elles le deviennent, bien souvent, suite à un présupposé de cohérence de l'ordre juridique qui anime le raisonnement judiciaire». Il ne s'agit pas ici de nier cette observation de bon sens. L'harmonie caractérise un système de loi idéal, et c'est cela même qui explique le "présupposé de cohérence juridique qui anime le raisonnement judiciaire ». Bien loin de négliger la pratique, le trait idéal-typique d'harmonie la justifie.

12. Le point de vue du magistrat, tel que je le définis, n'est donc pas strictement semblable, parce que plus concret, au point de vue de Dieu ou de «nulle part» dont nous entretient par exemple Thomas Nagel (1986). Il est bien plutôt le point de vue de Dieu soumis aux besoins de la régulation effective des comportements humains sublunaires et à ceux de la résolution de malentendus et de conflits interindividuels inévitables, comme on sait, depuis la Chute. 
pouvons entrer ici dans les minuties du débat entre vision positiviste et vision interprétative du droit ${ }^{13}$. Deux remarques suffiront. D'abord, le "point de vue des magistrats » n'est pas incompatible avec le rôle non automatique des juges en tant qu'interprètes de la loi. Distinguons sur ce point la logique de la justification légale et l'épistémologie du verdict. La "dérivation à partir d'une loi » décrit la logique de la justification. Sur le plan épistémologique, on rencontre des questions qui dépassent la syllogistique: comment établir ce qu'est « la loi » ? C'est là qu'intervient l'interprétation: lorsqu'un juge doit justifier une décision, il doit non seulement raisonner selon les formes, mais encore déterminer le contenu de la prémisse majeure ( «Il est interdit d'accomplir des actes de type $\mathrm{F} »)$. Et c'est cette opération qui appelle l'interprétation et ses mystères. Les problèmes herméneutiques, qui portent sur le contenu d'une prémisse, n'affectent en rien la vision syllogistique de la justification légale, qui porte sur la forme de l'argument. Si l'on s'interroge sur l'épistémologie du verdict - i.e. sur les conditions d'une décision particulière raisonnée -, alors l'interprétation et, plus généralement, l'identification de la loi ouvrent au juge une marge de manœuvre importante. Mais cela ne contredit en rien la thèse formelle selon laquelle des jugements non dérivés de la loi - mais d'une prémisse politique, morale, religieuse ou artistique inconnue des «sources de la loi» (codes, jurisprudence, doctrine) - ne sont pas des jugements légaux. Ensuite, au chapitre du positivisme, j'admets volontiers la thèse ontologique selon laquelle la loi est ce qui est contenu dans les sources de la loi ou qui est appliqué dans les tribunaux, ainsi que la thèse épistémologique selon laquelle pour connaître la loi, il faut observer - et peut-être interpréter - les sources et les pratiques juridiques. Cela réclamerait discussion; mais cette dernière nous entraînerait dans un examen approfondi des travaux de H. L. A. Hart et Joseph Raz, qui n'a pas sa place ici ${ }^{14}$. Cette forme de positivisme doit donc pour l'instant rester à l'état d'hypothèse plausible.

\section{Le point de vue éthique des gens de bien}

Le point de vue éthique est-il isomorphe au point de vue légal - celui des gens de bien soumis aux mêmes contraintes «informationnelles" que celui des magistrats? La question peut maintenant se poser avec plus de précision: est-il approprié, pour répondre à des questions éthiques, de se restreindre à des considérations normatives universelles, abstraites, impersonnelles, obligatoires, publiques, harmonieuses et explicites? On peut faire l'hypothèse d'un isomorphisme entre le point de vue des gens de bien et celui des magistrats. Les partisans classiques de la priorité libérale du juste sur le bien semblent l'admettre. Mais on peut aussi en douter. Je vais tenter, dans cette section, de réunir quelques-unes des très bonnes raisons connues de ne pas admettre l'hypothèse isomorphiste - parce que le point de vue légal exclut 
trop de considérations qui, dès lors qu'on jette un œil raisonnable sur les diverses manifestations réelles de la "vie éthique ", apparaissent d'une importance capitale. L'approche de l'éthique adoptée ici est analogue à la perspective jetée plus haut sur la loi: empirique et relativement proche du sens commun. Par "éthique ", j'entends ainsi l'ensemble des pratiques concrètes et observables qui se manifestent dans les efforts que les êtres humains font pour répondre, en paroles ou en actes, à la question de Socrate: "Comment doit-on vivre? ${ }^{15}$. La question qui nous occupe peut être ainsi reformulée: le point de vue légal est-il compatible avec nos pratiques éthiques -i.e. avec la (ou les) manière(s) dont nous tentons effectivement, dans nos "aventures " quotidiennes, de résoudre les problèmes qui soulèvent des questions impliquant, directement ou indirectement, celle de Socrate? Il existe de nombreuses raisons d'en douter. J'en évoquerai quelques-unes seulement.

\section{(i) Personnalité vs. impersonnalité, abstraction et généralité}

On peut commencer par noter que le point de vue du magistrat est inapproprié aux questions éthiques parce qu'il ignore l'importance de la personnalité. D'un point de vue impersonnel, les caractéristiques de l'agent éthique concret ne sont admises à influencer le jugement qu'à la condition d'être liées à des obligations spéciales, lesquelles sont créées par des actes eux-mêmes conformes à une règle générale - par exemple: «Si X promet à Y de faire $a$, alors $\mathrm{X}$ a l'obligation de faire $a$ ». Si la biographie de Jimmy contient une promesse faite à Max, cette dernière est reconnue par une règle générale et est donc admise, par le point de vue du magistrat, au titre d'information digne d'influencer le jugement. Mais hormis ce genre de traits abstraits explicitement spécifiés dans les règles elles-mêmes, tout détail concernant la biographie, la personnalité, les relations intersubjectives particulières de l'auteur d'un acte évalué doit être indifférent.

Or il existe de bonnes raisons de penser que le point de vue éthique requiert la prise en considération d'informations personnelles concrètes et particulières ${ }^{16}$. Il s'agit en éthique d'évaluer et de commenter des actes, des dispositions, des intentions, des caractères en général, ou des personnes («Max est généreux»). Concentrons-nous sur les actes.

a) Évaluer un acte, cela suppose qu'on l'ait d'abord compris. Il existe, c'est bien connu, pour toute séquence de mouvements physiques, un nombre indéterminé de "descriptions d'action» possibles. Si plusieurs descriptions d'action sont compatibles avec la même séquence de mouvements physiques, c'est que la description d'action contient plus que la

15. Cf. Williams 1985, chap. 1. Cette définition a le mérite d'être neutre à l'égard des grandes dichotomies théoriques - par exemple «conséquentialisme $v$ s. déontologisme». Elle est suffisamment vague, ce faisant, pour ne pas prédéterminer fallacieusement le résultat de la discussion à venir.

16. Cf. MacIntyre, 1984, Oakeshott, 1975, Taylor, 1989, et Williams, 1976. 
description de ces derniers ( $\mathrm{Il}$ a levé le bras droit et agité la main»). Quel est ce "plus»? L'intention. Il y a une relation interne entre cette dernière et la description d'action - c'est-à-dire que l'intention et l'action ne sont pas logiquement indépendantes. "Saluer de la main », c'est lever le bras et agiter la main dans l'intention de saluer de la main. Les mouvements physiques $m$ ne peuvent être décrits comme une action $a$ qu'à condition qu'on établisse un lien entre $m$ et une intention dont le contenu propositionnel comprend lui-même une description de $a$. Comprendre une action, dans la mesure où l'exercice requiert la sélection de la (ou d'une) bonne description d'action à appliquer aux mouvement physiques, cela demande donc qu'on découvre l'intention dans laquelle ils ont été exécutés (s'il y en a une). Mais cela demande plus encore. Supposons, comme le fait MacIntyre ${ }^{17}$, qu'un homme en aborde un autre et lui dise: «Le nom du canard sauvage commun est Histrionicus histrionicus histrionicus". Le second peut comprendre le sens de l'énoncé; mais peut-il comprendre l'acte (de langage) lui-même? Imaginons que le premier homme affirme, pour s'expliquer: "J'ai dit cela pour répondre à votre question "; l'autre: "Mais je ne vous ai posé aucune question»; le premier: "Je sais». Son acte demeure inintelligible. Nous comprenons le sens des mots qu'il a prononcés (définition scientifique usant de termes latins); nous connaissons son intention (répondre à une question); mais nous ne la comprenons pas. Puisqu'il $\mathrm{y}$ a une relation interne entre intention et description d'action, le doute portant sur la première est transféré sur la seconde. Pour comprendre l'intention, il faut avoir recours à des faits supplémentaires concernant l'agent. Par exemple: c'est un comique abordant les gens à l'improviste et leur disant des choses insolites pour provoquer des réactions amusantes. Son acte devient alors compréhensible - il peut être décrit ainsi: «Il m'a fait une farce pour une émission de télévision.» En d'autres termes, nous comprenons d'autant mieux une action que nous connaissons plus de faits concernant son auteur.

b) Cela est d'autant plus vrai que les actions à décrire sont complexes. Jusqu'ici, nous avons fait comme si les actions à comprendre constituaient des unités non problématiques. Mais l'individualisation des actes elle-même soulève des difficultés. Où commencent et où se terminent nos actes? Jimmy sonne à la porte de Max. Mais pourquoi ne pas dire: Jimmy accomplit l'avant-dernière phase de son plan pour assassiner Max? Ou: Jimmy vient rendre un disque à Max? Etc. La sélection du degré de généralité de la description d'action est elle aussi relative à l'histoire personnelle de l'agent, en ce sens qu'elle seule nous permet de déterminer si les mouvements physiques observés doivent être attribués à une intention ponctuelle ou à un plan s'étendant dans le temps 
et dont la réalisation suppose des enchaînements complexes d'actes subordonnés. L'individualisation des actions, elle aussi, suppose par conséquent des informations personnelles concernant l'agent et sa biographie.

Il ressort de cela que les considérations strictement impersonnelles et abstraites quant à la description de l'action ne sont pas suffisantes à la production d'un jugement éthique approprié. Pour qu'un jugement portant sur une action soit approprié, il faut que cette dernière ait été correctement et non arbitrairement individualisée, décrite et comprise - toutes opérations dont le succès requiert la connaissance d'informations personnelles concrètes et particulières. Illustrons cela par la discussion d'un exemple célèbre de règle universelle et impersonnelle, à savoir la véracité ou prohibition du mensonge, discuté par Kant dans son opuscule Sur le prétendu droit de mentir par humanité. Un ami est caché chez moi, qu'une milice brutale, illégitime et mal intentionnée recherche pour le mettre à mort. On sonne à ma porte; j'ouvre: les miliciens, qui demandent si je sais où il se cache. Dois-je mentir, ou doisje être véridique? L'universalisation de la "maxime de mon action" milite pour la seconde réponse. Mais décrire ainsi («mentir» et «être véridique») les deux «maximes » en balance est peut-être fallacieux. Si l'alternative était plutôt: dois-je être loyal envers mon ami; ou dois-je le trahir? Ou encore: dois-je empêcher des assassins de remplir leurs funestes desseins, ou les y aider? Dans ces deux cas-là, il est moins sûr que le test kantien d'universalisabilité accorde ses faveurs à la seconde option - laquelle paraît même un très absurde candidat au titre de corne d'un dilemme moral: il semble que toute personne raisonnable se doit d'opter «sans réfléchir » pour la première. Pourtant, cette nouvelle alternative contient des descriptions à première vue aussi adéquates de mon acte que l'alternative kantienne. L'argument de Kant, dans son opuscule sur le droit de mentir, frise la pétition de principe en ceci qu'il sélectionne, de manière passablement $a d$ hoc, certaines descriptions des actions ouvertes à l'agent qui prédéterminent le résultat du test. Kant choisit en effet de présenter son "dilemme ", qu'il prétend apparent, dans des termes impersonnels. Mais une description impersonnelle est-elle nécessairement plus fidèle aux enjeux éthiques soulevés par les situations concrètes de choix ? Par exemple, comment justifier que la description de mon action ne peut contenir le mot «ami» (et encore moins l'expression «mon ami»), qui est personnel en ceci qu'il suppose une certaine relation entre cet homme et moi, ainsi qu'une certaine part de biographie particulière en retraçant la naissance et en permettant de rendre compte de sa nature exacte? Le choix de la règle sous laquelle tombe l'action à évaluer suppose une certaine description de l'action, laquelle peut-être biaisée - ici, en faveur de la véracité. Préférer la première formulation de l'alternative à la seconde, cela revient à accorder une priorité à la véracité sur l'amitié. Nous voyons ainsi que le choix du degré de généralité et d'abstraction de la description des actions à évaluer est luimême une question éthique que la focalisation sur l'impersonnalité des règles 
universelles ne permet pas de résoudre impartialement ${ }^{18}$. Il nous faut pour cela faire appel à des informations personnelles et biographiques choisies avec soin - lesquelles peuvent alors nous entraîner à opter pour une description personnelle des actions et donc pour une considération prescriptive elle-même personnelle ( Max n'a pas le droit de faire ça à Jimmy») ${ }^{19}$.

\section{(ii) Particularité vs. généralité et abstraction}

Ce qui vaut pour la compréhension et l'individualisation des actions vaut également pour la description des situations de choix. Rappelons ici ce que Stuart Hampshire nomme l'inexhaustibilité de la description ${ }^{20}$. L'inexhaustibilité peut recevoir la description suivante: primo, "toute situation à laquelle je suis confronté, et qui n'est pas une situation de jeu, a un ensemble inépuisable de caractéristiques discriminables allant au-delà de celles que je remarque explicitement sur l'instant parce qu'elles sont pour moi d'un intérêt immédiat ${ }^{21}$; secundo, la situation peut posséder des caractéristiques auxquelles il est difficile de faire référence au moyen du vocabulaire que j'emploie ${ }^{22}$. Or, du point de vue légal, les critères de spécification doivent eux-mêmes être gouvernés par des règles juridiques - comme en atteste la qualification des circonstances atténuantes (délit passionnel, irresponsabilité) -, sans quoi les

18. Cela implique qu'il existe des descriptions éthiquement supérieures à d'autres. On peut en douter, mais l'exemple suivant devrait suffire à dissiper l'embarras. Rappelons-nous le célèbre dilemme moral raconté par Hermann Melville dans Billy Budd, marin. Un homme foncièrement bon en tue involontairement un autre, foncièrement mauvais, à la suite d'une escalade de persécutions du premier par le second - et ce, sur un vaisseau de la marine de guerre britannique dont les règlements punissent ce type d'actes de la peine de mort. Le capitaine du navire et quelques officiers réunis en «conseil » doivent juger que faire, puisqu'il y a eu infraction. Le noble "Vere", capitaine, que nous présente Melville a conscience d'être face à un dilemme. Il décrit la situation comme un dilemme: le «meurtrier» involontaire est foncièrement bon et innocent; mais l'acte accompli est indéniablement un crime condamné par la loi militaire. Respecter la loi ? Ou, privilégiant l'évaluation éthique de la situation, acquitter le coupable? Le Vere de Melville fera condamner Budd. Comparons-le avec un autre capitaine, lequel serait parvenu au même jugement (la corde) sans pourtant éprouver la moindre angoisse tragique, parce qu'il ne voit la situation qu'ainsi: Billy Budd a violé la loi militaire - oubliant le versant «mystérieux", comme l'écrit Melville, de toute l'affaire. Auquel des deux capitaines fictifs va notre "préférence éthique" ? À Vere, bien entendu. Mais la seule différence le distinguant de son double est qu'il perçoit la complexité de la situation. Cet exemple intuitif semble donc attester que le choix d'une description de la situation de choix est lui-même une question éthique. Pour une excellente analyse de cette question dans une ligne aristotélicienne, cf. Sherman (1989): 29 sqq.

19. Pour d'autres arguments allant dans ce sens, cf. par exemple Wiggins (1987).

20. Cf. Hampshire, 1977 et 1979.

21. Hampshire, 1977, 30.

22. Ibid. Il se peut qu'un acte que je me propose d'accomplir vaille, pour le membre d'une culture éloignée de la mienne qui serait "partie prenante" de la situation de choix, comme un très blessant manque de respect que mon vocabulaire m'interdit de décrire comme tel - parce qu'il fait appel à une notion morale «épaisse " que ma culture ignore (impliquant par exemple une référence à l'acte de «saluer une tierce personne de la main lorsqu'on est en train de converser pour la première fois avec un aîné"). 
jugements légaux ne peuvent plus prétendre à l'incontestabilité nécessaire au rôle qu'ils doivent remplir dans la résolution des disputes et des ambiguités. Les règles juridiques, par conséquent, doivent présélectionner les traits spécifiques pertinents appelés à entrer dans les descriptions d'action - elles doivent fixer un ensemble épuisable de traits pertinents à la description des actes qui tombent sous leur juridiction. D'où il suit qu'elles ne peuvent prendre en considération la nouveauté imprévisible, le caractère souvent insolite et l'ambiguité propres aux situations concrètes de choix. Comme nous l'avons déjà vu, le choix des descriptions - et donc des propriétés d'une action, d'un agent ou d'une situation concrets - éthiquement pertinentes est lui-même une question éthique que les règles tranchent avant que les objets concrets de la description n'apparaissent et puissent être soumis à l'examen. On n'est jamais sûr, par voie de conséquence, que les critères de spécification prévus par les règles juridiques ne laissent pas échapper certaines différences particulières irréductibles qui peuvent, pourtant, s'avérer d'une importance capitale pour le jugement éthique.

L'importance de la description des actions et des circonstances particulières pour l'obtention d'un jugement éthique approprié suggère un modèle de la rationalité éthique passablement aristotélicien, dans lequel le discernement des particuliers joue un rôle indispensable et peut-être suffisant ${ }^{23}$. On peut modeler ce discernement sur une analogie entre jugement éthique et identification perceptive ${ }^{24}$, ou sur une analogie entre jugement éthique et «jugement de goût » auquel on parvient par l'exercice d'un savoir-faire implicite appris par imitation, exploration et trial and error ${ }^{25}$. Dans les deux cas, on doit insister sur le rôle de l'expérience dans l'acquisition et l'affinement du discernement. Mais je m'abstiendrai, faute de place, d'examiner ici en détail la question - qui touche à des problèmes aussi vastes que la justification non-doxastique ou la signification des concepts éthiques. Notons seulement que ces hypothèses aristotéliciennes et humiennes suggèrent un point de vue éthique semblable, plutôt qu'à celui du magistrat, à ceux de l'esthète ou de l'homme de goût, lesquels permettent d'arriver à des jugements appropriés au moyen de "procédures" dans lesquelles l'universalité, l'impersonnalité, l'abstraction et l'explicite n'interviennent guère - ne sont pas nécessaires.

\section{(iii) Conflit vs. harmonie}

Admettons donc l'importance des informations particulières, concrètes et personnelles dans l'obtention d'un jugement éthique approprié. Il ne s'agit pas de nier ici que l'éthique ait, d'une manière ou d'une autre, maille à partir avec l'impersonnalité ou l'impartialité - ce serait une manœuvre kamikaze.

23. Cf. Aubenque, 1962, Hampshire, 1977 et 1979; Nussbaum, 1990, chap. 2, 54-105; Reeve, 1992, 67-73; Sherman, 1989, chap. 2, 13-55).

24. Cf. Hampshire, 1977 et 1979, Murdoch, 1956 et 1970.

25. Cf. Hampshire, 1979, et Baier, 1985, chap. 11 et 12, 207-227, 228-245. 
Jusqu'ici, j'ai soutenu que le point de vue éthique se distinguait du point de vue légal en ceci qu'il est souvent nécessaire, face aux questions éthiques, de tenir compte d'informations particulières et personnelles. Cela n'implique que pas les considérations universelles (ou générales) n'ont aucun rôle éthique à jouer; cela implique, en revanche, qu'il y a une source perpétuelle de conflits éthiques possibles: ceux qui, toujours, peuvent survenir entre les considérations impersonnelles (quel que soit leur rôle précis) et les considérations personnelles. La question du droit de mentir par humanité en est une illustration.

Nous rencontrons ici une autre critique majeure souvent adressée à l'hypothèse d'une identité des points de vue éthique et légal, qui consiste à revendiquer le caractère insuppressible des conflits éthiques. Considérons l'expression suivante: "Jimmy a commis un vol généreux » - ou «Jimmy a volé généreusement». Elle exprime à n'en pas douter un jugement éthique, d'un type assez ordinaire, qui inclut la qualification adverbiale d'une action. L'action de Jimmy, dans notre exemple, est décrite à la fois comme la violation d'une règle juridique - la prohibition du vol - et comme la manifestation d'une vertu. Il s'agit d'une action ambiguë. Mérite-t-elle le blâme ou l'approbation? Pour en décider, il faut balancer l'une contre l'autre légalité et générosité: quelle considération devait donc l'emporter ? Et nous voilà face à un conflit éthique.

C'est une "donnée de l'éthique » - à savoir un fait qu'on peut observer quand on examine les manifestations ordinaires de la vie éthique et les infinies conversations dont elle se compose en bonne part - que lorsqu'il s'agit d'évaluer des actes (ou des caractères), on donne (parfois, souvent) de ces derniers des descriptions éthiquement ambiguës. Ces dernières reposent en grande partie sur la possibilité des qualifications adverbiales de l'action. Les descriptions ambiguës peuvent à leur tour donner lieu à des conflits éthiques. Si, comme Billy Budd, j'ai innocemment tué quelqu'un, mon cas peut aussi bien appeler un jugement positif que négatif. Nous avons déjà noté que les systèmes juridiques supportent mal les verdicts équivoques, car ces derniers sont sujets à contestation ultérieure. Le point de vue des magistrats exclut les jugements ambigus - et partant les considérations mutuellement conflictuelles. Or, pour ce faire, il doit également exclure les descriptions d'action adverbiales, qui ouvrent la possibilité de l'ambiguïté et du conflit. Mais ces dernières semblent bien faire indissolublement partie de nos pratiques éthiques - et donc du point de vue des gens de bien.

\section{(iv) Évocation vs. reconnaissance publique}

Je serai plus bref pour les deux dernières remarques qu'il me reste à présenter. Nous avons déjà admis que les considérations éthiques ne pouvaient être strictement impersonnelles et abstraites, et que le souci de porter des jugements éthiques justifiés milite en faveur d'une attention scrupuleuse aux particularités. Cela ouvre la possibilité suivante: certains traits éthiquement pertinents dans la description de la situation de choix et des actions évaluées 
peuvent être tellement insaisissables qu'il est impossible de leur donner une formulation susceptible d'être publiquement comprise et reconnue. Un trait pertinent peut être tellement particulier, justement, qu'il manque au langage que je possède une expression adéquate pour le décrire. Reste le recours à des paraboles, à des métaphores, à des comparaisons - bref, à des «techniques d'évocation» tellement idiosyncratiques qu'elles sont inaptes à faire l'objet d'une discussion publique de type légal ${ }^{26}$ : "Elle avait l'âme oblique comme une paupière javanaise » (Henry Miller) - tel pourrait être, par exemple, le genre de description, "non scientifique ", indispensable à mon appréciation du caractère d'autrui. La reconnaissance publique, pourtant, est une condition nécessaire des règles juridiques et des considérations admises à influencer les jugements légaux. Or les considérations «évocatrices» sont par trop ouvertes à la diversité des interprétations pour figurer dans les débats publics auxquels doivent être soumis les jugements légaux. Une fois encore, nous voyons naître le soupçon tenace que le point de vue du magistrat est trop restrictif pour traiter des questions éthiques.

\section{(v) Subérogation et superérogation vs. obligation}

Enfin, rappelons-nous que le point de vue légal n'admet, au rang des « raisons d'agir », que des obligations - à savoir des prescriptions dont l'ignorance entraîne nécessairement des sanctions formelles ou informelles. Or il est connu que les questions éthiques font parfois appel à des considérations qui vont au-delà de l'obligation ou qui restent en deçà. Si un enfant est prisonnier d'une maison en flammes, j'ai une raison éthique de me porter à son secours au mépris de ma sécurité, - mais on ne me sanctionnera pas si je demeure immobile. Les actions héroïques sont superérogatoires : elles demandent trop de sacrifices potentiels à l'agent pour être obligatoires. Si un mendiant me réclame une obole, j'ai une raison éthique de lui répondre aimablement (par exemple parce que Jésus a dit: "Aime ton prochain»), - mais on ne me sanctionnera pas si je lui crache un mot d'insulte. Les actions aimables sont subérogatoires: leur accomplissement ne promeut pas un intérêt d'autrui qui soit suffisamment «vital » pour être obligatoire. Par conséquent, le point de vue légal exclut des considérations non obligatoires qui sont pourtant partie intégrante de nombreux jugements éthiques ${ }^{27}$.

\section{Le paternalisme moral}

Il faut se garder d'assimiler le point de vue des gens de bien au point de vue des magistrats. Ce dernier, en effet, impose aux considérations admises à influencer le jugement des contraintes trop strictes pour que soient prises en considération certaines complexités inhérentes à nos manières, non pas de théoriser sur l'éthique, mais, pour ainsi dire, de la pratiquer - au jour le jour,

26. Cf. Murdoch, 1970 et Nussbaum, 1990.

27. Cf. Williams, 1985, chap. X, 188-211. 
dans une infinie diversité de situations et de contextes auxquels nous répondons de façon parfois ambiguë et auxquels nous devons sans cesse nous adapter. L'éthique est souple; la loi est rigide. De même que les critères (esthétiques) d'évaluation littéraire sont souples - pour cette raison qu'on ne peut les codifier explicitement sous la forme de règles absolues et universelles -, tandis que les critères d'évaluation orthographique ou grammaticale sont rigides. À celui qui déclarerait que Céline est un mauvais écrivain sous prétexte qu'il «bousille la grammaire française » et que Jean d'Ormesson est un génie de la plume sous prétexte qu'il respecte toutes les règles inventoriées dans le Grévisse, nous répondrions qu'il porte un jugement littéraire inapproprié parce que basé sur des considérations trop étroites ${ }^{28}$. Si la discussion qui précède est convaincante, il faudrait adresser le même reproche à celui qui jugerait que Jimmy est un monstre sous prétexte, uniquement, qu'il n’a "aucun respect pour les lois universelles".

Si nous acceptons ce qui précède, il s'ensuit que «la bonne chose à faire " dans une situation de choix particulière est une fonction complexe de facteurs imprévisibles, parfois conflictuels et rétifs aux descriptions explicites et propres à la discussion publique. Par conséquent, "la bonne chose à faire » dans une circonstance particulière dépend de facteurs qui échappent nécessairement au point de vue légal. Au mieux, ce dernier peut nous dire que, dans une situation de type $F$, les actes de type $G$ sont prohibés ou obligatoires. Mais il demeure une possibilité éthique - à savoir que dans cette situation de type $\mathrm{F}$ où $j e$ suis immergé maintenant, "la bonne chose à faire " soit précisément un acte de type G. Il se peut par exemple que mon accomplissement d'un acte de type $G$ possède, en raison d'une propriété singulière de cette situation de type $\mathrm{F}$ qu'il serait impossible de prévoir ni même de décrire en termes abstraits, une propriété elle-même singulière qui en fasse «la bonne chose à faire ${ }^{29}$. Le point de vue des magistrats ne le condamnerait pas moins pour autant ${ }^{30}$.

La distinction entre point de vue des magistrats et point de vue des gens de bien jette ainsi une lumière intéressante sur le paternalisme. Ce dernier a toujours figuré parmi les cibles de choix du libéralisme - de la Lettre sur la tolérance de Locke à nos jours, en passant par De la liberté de Mill. Le paternalisme qui nous intéresse ici n'est qu'une variété du paternalisme en

28. À supposer, bien entendu, que la correction grammaticale ait quoi que ce soit à voir avec la qualité littéraire - ce qui est loin d'être sûr.

29. Tel est peut-être, par exemple, le message méta-éthique du dernier film de Clint Eastwood, Million Dollar Baby.

30. D'où les affres de Javert: «Il se disait que c'était donc vrai, qu'il y avait des exceptions, que l'autorité pouvait être décontenancée, que la règle pouvait rester court devant un fait, que tout ne s'encadrait pas dans le texte du code, que l'imprévu se faisait obéir, que la vertu d'un forçat pouvait tendre un piège à la vertu d'un fonctionnaire, que le monstrueux pouvait être divin, que la destinée avait de ces embuscades-là, et il songeait avec désespoir que lui même n'avait pas été à l'abri d'une surprise» (Hugo 1862, vol. 3, 382). 
général. Communément, est dit "paternaliste» tout projet de faire le bien d'autrui si nécessaire contre son gré. Le paternalisme prudentiel consiste ainsi à vouloir faire le bien non moral des gens, si nécessaire contre leur gré (dans les domaines de la santé ou de la sécurité routière, par exemple). Par contraste, le paternalisme moral consiste à vouloir faire le bien moral des gens, si nécessaire contre leur gré. De quelque manière qu'on définisse le «bien moral », il s'agit dans cette perspective de contraindre les gens à être, non pas seulement de bons partenaires non violents de la coopération sociale - de bons citoyens -, mais aussi des agents moralement droits - de «bons chrétiens». Le paternalisme moral qui nous intéresse est en outre un paternalisme de la loi, dans lequel l'instrument contraignant envisagé est l'obligation légale appuyée par la police et appliquée par des tribunaux. Il se manifeste notamment dans l'efflorescence de projets de loi visant à punir la «violence psychologique», l' "insulte» ou diverses catégories d' «incivilités» - afin de chasser par la force l'immoralité du monde. Le paternalisme moral nous dit donc: si l'acte $\mathrm{X}$ est moralement condamné, il doit être légalement interdit; s'il est moralement impératif, il doit être légalement obligatoire; et, dans les limites du possible, il faut s'efforcer de doubler le réseau des devoirs moraux d'un réseau de lois et de sanctions. Moins discuté dans la littérature que la variante prudentielle, le paternalisme moral n'en est pas moins indéniablement à la mode dans les «opinions publiques» contemporaines.

Comment évaluer le paternalisme moral à la lumière de la distinction entre points de vue présentée plus haut? Empruntons à Joseph Raz ${ }^{31}$ une caractérisation expédiante de la "justification normale de l'autorité »: «La manière normale d'établir qu'une personne a une autorité "justifiée" sur une autre personne implique que l'on montre que le sujet putatif est susceptible de mieux obéir aux raisons qui s'appliquent à lui [...] s'il accepte les directives de l'autorité putative comme légitimement contraignantes et s'il essaie de s'y plier, plutôt que s'il tentait d'obéir aux raisons qui s'appliquent à lui directement. "Supposons que j'aie une raison de ne pas être violent avec mes voisins (par exemple parce que Dieu l'interdit). Si, en suivant vos directives, je suis susceptible de mieux obéir (plus souvent, plus strictement) à cette raison qui s'applique à moi, alors il est normalement justifié que vous jouissiez de l'autorité de contrôler la non-violence de mon comportement. Nous constatons bientôt que le paternalisme moral ne passerait pas le test. Les « directives de l'autorité putative », en effet, dès lors qu'elles respectent l'autorité de la loi et dès lors, partant, qu'elles s'inscrivent dans un système juridique ressemblant peu ou prou à celui que j'ai décrit plus haut, ne peuvent jamais être entièrement fidèles aux "raisons » éthiques qui s'appliquent concrètement $\mathrm{au}$ «sujet putatif» dans ses tribulations et délibérations réelles. Pourquoi ? Parce que les «directives de l'autorité putative», dans un État «rationnel bureaucratique " moderne soumis à l'autorité de la loi, doivent s'adapter au

31. Joseph Raz, 1986, 53. 
«format» prévu par le point de vue du magistrat. Si je reconnais l'autorité de $\mathrm{X}$ dans un certain domaine, je considère, comme le rappelle $\mathrm{Raz}^{32}$, que ses commandements dans ce domaine sont contraignants "même s'ils sont perdants sur la balance des raisons ». En cas de conflit moral entre deux options $a$ et $b$, si la loi prescrit $a$, je serai obligé de privilégier $a$-même si en pesant le pour et le contre en toute conscience je pense que $b$ est dans ce cas précis la raison la plus forte. Reconnaître l'autorité de la loi dans le domaine de la poursuite du bien, c'est soustraire des directives impersonnelles, abstraites, générales, harmonieuses et explicites à la balance des raisons en leur conférant une force par principe «imbattable». Ce qui revient à soumettre ses délibérations éthiques à un format qui leur est impropre. Partant, le sujet putatif n'est pas plus «susceptible» de mieux obéir aux « raisons qui s'appliquent à lui» s'il confie à la loi l'autorité de promouvoir le bien. L'autorité de l'État et des tribunaux (du système juridique) dans le domaine éthique de la quête du bien ne saurait donc recevoir une justification «normale».

Plusieurs objections sont ici possibles. Premièrement, on peut invoquer la casuistique des circonstances atténuantes et la marge de manœuvre discrétionnaire des juges, qui permettraient de faire place, à l'intérieur du point de vue légal, à la prise en considération des «anomalies» particulières. Mais quelles que soient par ailleurs les circonstances d'un vol, celui-ci n'en reçoit pas moins, du point de vue légal, la valeur négative d'une infraction qu'il faut punir (ou du moins réprimander). En outre, même un juge perspicace ne peut connaître les circonstances particulières d'un acte mieux que les agents directement immergés dans la situation de choix. Tenez compte de toutes les circonstances atténuantes prévisibles par un système juridique et de toute la perspicacité des juges, vous ne pourrez néanmoins parvenir au jugement éthique que le vol avait une valeur positive. Or c'est précisément cette possibilité dont les arguments présentés plus tôt tentaient de démontrer qu'elle était nécessaire à la réflexion et à l'expérience éthiques. Nos deux points de vue sont décidément irréductibles. Deuxièmement, on peut proposer d' "assouplir » le système juridique lui-même. Cela peut vouloir dire deux choses. Soit il s'agit de multiplier les clauses spécifiques garantissant la malléabilité des jugements légaux: nous venons de voir les faiblesses de cette tactique. Soit il s'agit de supprimer le système juridique lui-même et de donner aux responsables de l'emploi de la contrainte publique une marge de manœuvre soustraite au contrôle légal. Il existe des arguments politiques indépendants - en faveur de l'autorité de la loi -, qu'on connaît bien et sur lesquels je ne m'étends donc pas, pour suggérer que ce serait une fort mauvaise idée si l'on tient à garantir la stabilité et la paix civiles nécessaires à la coopération sociale. Troisièmement, on peut revenir sur le fait qu'il semble exister des règles universellement reconnues et appliquées, comme la prohibition du meurtre ${ }^{33}$. Le meurtre est moralement interdit; et il

32. Raz, 1990, 62-65.

33. Encore que... Pour l'expression convaincante de quelques doutes à ce sujet, cf. Baier (1985, chap. 11, 207-227). 
est légalement interdit. Quel est le lien entre ces deux prohibitions: la première justifie-t-elle la seconde - le meurtre est-il légalement interdit parce qu'il est moralement interdit? C'est loin d'être certain. La prohibition légale du meurtre n'a pas besoin du paternalisme moral pour être justifiée: elle peut s'appuyer, tout aussi bien, sur les nécessités sociales et politiques de la vie en commun plutôt que sur les nécessités d'une vie individuelle bonne ${ }^{34}$. On peut ainsi lui donner une justification prudentielle classique: nous avons tous intérêt à la paix civile et à la coopération; l'État est le meilleur garant de ces deux biens "égoïstes "; donc, l'État se caractérisant par le monopole de la violence légitime, et le meurtre étant une forme de violence incompatible avec ce monopole, nous avons intérêt à ce que le meurtre soit légalement interdit. La prohibition légale du meurtre peut recevoir des justifications non morales. En outre, le besoin de sécurité est une des raisons majeures d'accepter l'autorité de l'État, et la protection contre le meurtre est un élément central de la sécurité. L'État, par le biais de la loi, interdit donc le meurtre avec l'accord de tous les esprits raisonnables. La prohibition légale du meurtre n'a donc pas non plus besoin d'une justification paternaliste. C'est pourquoi le rejet du paternalisme moral n'est aucunement incompatible avec l'adhésion à certaines prohibitions légales fondamentales (du meurtre, du viol, du vol).

Bref. La conclusion s'impose enfin: la résolution optimale des questions éthiques requiert que le point de vue éthique soit clairement distingué du point de vue légal. D'où il suit que la loi ne peut en rien réclamer l'autorité d'exercer sur celles-là son pouvoir décisionnel contraignant. Il est donc éthiquement recommandé de rejeter le paternalisme moral. Faute de quoi, on risquerait d'emprisonner les «mollusques éthiques» que nous sommes, non pas uniquement dans une "carapace morale", comme dirait Robert Musil, mais dans un cercueil légal. Un cercueil légal est plus rigide qu'une simple coquille, et plus artificiel, pour la simple raison que ses parois ont la dureté de tout ce qui a trait à l'emploi du monopole de la violence légitime et à l'opération des appareils bureaucratiques. Le paternalisme moral reviendrait ainsi à substituer au jugement éthique individuel un jugement moralisateur déformé par le point de vue légal - et, peut-être, à encourager une nouvelle forme de vice: le pharisaïsme de la matraque.

\section{Conclusion}

Tout législateur sensé et conscient de l'hétéromorphisme entre le point de vue des gens de bien et le point de vue des magistrats devrait ainsi nourrir, à l'égard du paternalisme moral, un scepticisme de marbre: pour des raisons

34. J’appelle «nécessités de la vie en commun» les besoins de coopération et de coordination qu'ont les individus vivant en communauté (plus ou moins étendue). Il importe peu, sur ce plan, de savoir à quelles fins individuelles vont concrètement servir la coopération et la coordination, l'essentiel étant que les conditions soient réunies qui permettent la poursuite des fins individuelles - conditions au nombre desquelles il faut mentionner la sécurité et la possibilité de nourrir des attentes à long terme concernant le comportement d'autrui. 
méta-éthiques, il est contre-indiqué de rendre l'exercice des vertus légalement obligatoire. Auquel cas le philosophe politique - s'il s'interroge, du point de vue "constitutionnel ", sur les formes justifiées d'un système de droit et de justice - a une excellente raison d'arriver à une conclusion libérale (parce qu'anti-paternaliste). Certes, il est justifié que la loi interdise le meurtre ou le vol; et, certes, toutes les théories morales crédibles interdisent elles aussi le meurtre ou le vol. Il y a ainsi sur ces points, entre "loi » et "morale », une indéniable coïncidence. Mais de même que, comme on le sait dans les sciences sociales, corrélation n'est pas causalité, de même, en philosophie normative, coïncidence n'est pas justification. Certes, j'ai de bonnes raisons d'accorder au magistrat l'autorité d'interdire le meurtre. Mais comme nous l'avons vu plus haut, ces raisons peuvent être strictement prudentielles: je dois obéir à la loi, dans le domaine des homicides et des vols, non pas parce que j'ai l'interdiction morale de tuer ou de voler, mais parce que j'ai un intérêt égoïste à n'être ni assassiné ni dépouillé. La justification normale de l'autorité passe bien, ici, par des raisons non morales. La loi peut donc trouver un contenu justifié même si l'on rejette la logique du paternalisme moral.

Se dessine ainsi une forme de libéralisme "vulgaire " résistant à la tentation de revendiquer, pour contrer les objections communautaristes, une forme perfectionniste de la justification politique ${ }^{35}$. Car le point de vue normatif n'existe pas et se disperse en points de vue moins généraux commandant des justifications hétéromorphes. Il est donc vain de vouloir calquer le point de vue adapté aux problèmes politiques de bonne organisation de la vie commune sur le point de vue adapté aux problèmes de bonne conduite de la vie individuelle. Contrairement à ce que pense la compagnie des philosophes post-rawlsiens, il est faux de soutenir que «la philosophie politique [...] relève de l'argumentation morale ${ }^{36}$. Puisque la philosophie politique s'intéresse à la bonne organisation de la vie en commun, et puisque la vie en commun fait nécessairement intervenir des règles de droit, la philosophie politique doit tenir compte de contraintes justificatives plus strictes que ne le réclament nos investigations éthiques en général.

À tout le moins, l'affaire est donc moins simple que ne le suggère Kymlicka. L'hypothèse d'une continuité entre éthique et politique est contestable. Les arguments employés plus haut pour montrer l'importance du particulier en éthique sont pour la plupart issus d'un courant largement antikantien - et parfois même empruntés à MacIntyre lui-même. Et pourtant, ils entrent dans une défense libérale de la neutralité de la loi en matière de promotion du bien. Il n'est donc aucunement besoin d'être kantien pour être libéral. On peut nier en éthique la priorité sur le bien des règles générales et impersonnelles de la justice et pour cette raison même adopter des positions politiques libérales (en l'occurrence l'antipaternalisme éthique et la neutralité

35. Cf. Neal, 1993.

36. Kymlicka, 1992, 14. 
de l'État) - parce que le bien n'est pas le genre de chose que la loi peut promouvoir et que cette dernière doit se cantonner à remplir un rôle plus humble, mais à sa mesure. Par conséquent, comme il était suggéré dans l'introduction, si les communautariens comme MacIntyre ont raison (en éthique), alors les libéraux ont raison (en politique). Mais si Kant a raison, il semblerait que les libéraux aient aussi raison (à en croire les communautariens). Faut-il conclure que le libéralisme est à tel point fidèle à ses aspirations, et donc tellement neutre en ce qui concerne le bien, qu'on doive y arriver quelle que soit la théorie éthique qu'on prend pour point de départ? Voilà qui est peutêtre vulgaire, mais certainement robuste.

\section{Bibliographie}

Aubenque, P. La prudence chez Aristote, Presses universitaires de France, coll. "Quadrige", 1962.

Baier, A. Postures of the Mind. Essays on Mind and Morals, Methuen, 1985.

Braithwaite, R. B. "Moral Principles and Inductive Policies", in Findlay J. N. éd. (1966), Studies in Philosophy. British Academic Lectures, Oxford University Press, 1966.

Gaus, G. F. «Reasonable Pluralism and the Domain of the Political: How the Weaknesses of John Rawls's Political Liberalism Can be Overcome by a Justificatory Liberalism ", Inquiry, 42, p. 259-284, 1999.

Hampshire, S. Two Theories of Morality, Oxford University Press, 1977.

—. "Public and Private Morality", in Hampshire éd. (1979), Public and Private Morality, Cambridge University Press, 24-53, 1979.

Hart, H. L. A. Le concept de droit, 1961, trad. de l'anglais M. Van De Kerchove, Publications des facultés universitaires Saint-Louis, 1976.

Hugo, V. Les Misérables (1862), 3 vol., Gallimard, coll. «Folio », 1973.

Kant, E. Critique de la raison pratique (1788), trad. de l'allemand L. Ferry et H. Wismann, Gallimard, coll. «Folio», 1985.

Kekes, J. Against Liberalism, Cornell University Press, 1997.

Kelly, J. M. A short History of western legal Theory, Oxford University Press, 1992.

Kymlicka, W. Les théories contemporaines de la justice. Une introduction (1990), trad. fr. M. St-Opery, La Découverte 1999.

Locke, J. Lettre sur la tolérance et autres textes (1667), éd. J.-F. Spitz, GarnierFlammarion, 1992.

Macedo, S. «The Politics of Justification» (1990), Political Theory, vol. 18, n 2 (mai 1990), 280-304.

Macintyre, A. After Virtue, Notre Dame, 1981.

Murdoch, I. «Vision and Choice in Morality»(1956), in Murdoch, 1997, 76-98.

—. La souveraineté du bien (1970), trad. de l'anglais C. Pichevin, éd. de l'Éclat, 1994.

—. Existentialists and Mystics. Writings on philosophy and literature, éd. P. Conradi, Penguin, 1997.

Musil, R. L'homme sans qualités, trad. de l'allemand Ph. Jacottet, Seuil, coll. «Points », 1956.

Nagel, T. Le point de vue nulle part (1986); trad. de l'anglais S. Kronlund, éd. de l’Éclat, 1993. 
- "Moral Conflict and Political Legitimacy", Philosophy and Public Affairs, vol. 16, n 3 (été 1987), 215-240.

Neal, P. "Vulgar Liberalism », Political Theory, vol. 21, n 4 (nov. 1993), 623-243.

Nussbaum, M. Love's Knowledge. Essays on Philosophy and Literature, Oxford University Press, 1990.

Oakeshott, M. On human Conduct, Oxford University Press, 1974.

Raz, J. The Morality of Freedom, Oxford University Press, 1986.

—. Practical Reason and Norms, Princeton University Press, 1990.

Reeve, C. D. C. Practices of Reason. Aristotle's Nicomachean Ethics, Oxford University Press, 1992.

Renaut, A. Libéralisme politique et pluralisme culturel, Éditions Pleins Feux, 1999.

Sherman, N. The Fabric of Character. Aristotle's Theory of Virtue, Oxford University Press, 1989.

Skorupski, J. "Liberty's Hollow Triumph », in Ethical Explorations, Oxford University Press, 1999, chap. XI., 234-254.

Taylor, Ch. Sources of the Self. The Making of the Modern Identity, Harvard University Press, 1989.

Wacks, R. Understanding Jurisprudence, Oxford University Press, 2005.

Wiggins, D. "Universalizability, Impartiality, Truth ", in Needs, Values, Truth. Essays in the Philosophy of Value, Basil Blackwell, 1987, 59-86.

Williams, B. «Personne, caractère et morale» (1976), in Williams, 1994, 227-51.

- L'éthique et les limites de la philosophie (1985), trad. de l'anglais M.-A. Lescourret, Gallimard, 1990.

—. La fortune morale. Moralité et autres essais, choix de textes et trad. de l'anglais J. Lelaidier, Presses Universitaires de France, 1994. 